\title{
Numerical investigation of the effect of substrate surface roughness on the performance of zigzag graphene nano- ribbon field effect transistors symmetrically doped with BN
}

\author{
Majid Sanaeepur ${ }^{*}$, Arash Yazdanpanah Goharrizi and Mohammad Javad Sharifi
}

\author{
Full Research Paper \\ Address: \\ Department of Electrical and Computer Engineering, Shahid Beheshti \\ University, Velenjak Ave., Tehran, Postal Code: 1983963113, Iran

\section{Email:} \\ Majid Sanaeepur ${ }^{*}$ - m_sanaee@sbu.ac.ir \\ * Corresponding author

\section{Keywords:} \\ boron nitride; non-equilibrium Greens function (NEGF); on-/off-current \\ ratio; substrate roughness; zigzag graphene nanoribbon field effect \\ transistor (ZGNRFET)
} \author{
doi:10.3762/bjnano.5.168 \\ Received: 04 June 2014 \\ Accepted: 03 September 2014 \\ Published: 17 September 2014 \\ Associate Editor: R. Naaman
}

Beilstein J. Nanotechnol. 2014, 5, 1569-1574.

(C) 2014 Sanaeepur et al; licensee Beilstein-Institut.

License and terms: see end of document.

\begin{abstract}
The performance of field effect transistors comprised of a zigzag graphene nanoribbon that is symmetrically doped with boron nitride (BN) as a channel material, is numerically studied for the first time. The device merit for digital applications is investigated in terms of the on-, the off- and the on/off-current ratio. Due to the strong effect of the substrate roughness on the performance of graphene devices, three common substrate materials $\left(\mathrm{SiO}_{2}, \mathrm{BN}\right.$ and mica) are examined. Rough surfaces are generated by means of a Gaussian auto-correlation function. Electronic transport simulations are performed in the framework of tight-binding Hamiltonian and non-equilibrium Green's function (NEGF) formalisms. The results show that with an appropriate selection of the substrate material, the proposed devices can meet the on/off-current ratio required for future digital electronics.
\end{abstract}

\section{Introduction}

Field effect transistors (FETs) with a $10 \mathrm{~nm}$ gate length are stipulated by the International Technology Roadmap for Semiconductors (ITRS) for the year 2020 [1]. Regarding the Si scaling limits, it is obvious that for device lengths to be scaled below $10 \mathrm{~nm}$ new materials and device concepts are inevitable. Graphene is one of the most promising materials as a substitute for Si nanotransistors [1,2]. Due to its remarkable electronic properties, graphene has attracted significant attention from physicists and device engineers $[3,4]$. Unmodified graphene sheets do not have a band gap, therefore graphene transistors are not suitable for digital applications for which a minimum band gap of $360 \mathrm{meV}$ is needed [5,6]. Nevertheless, graphene nanoribbons (GNRs) smaller than $15 \mathrm{~nm}$ show a bandgap inversely proportional to the width of the GNR [7-11]. Armchair graphene nanoribbons (AGNRs) are non-magnetic. Zigzag graphene nanoribbons (ZGNRs), however, have a spin- 
polarized ground state and a high density of states localized at the zigzag edges of the ribbon [8,12-14]. Nonetheless, the energy of the spin-polarized state is only $20 \mathrm{meV}$ per edge atom lower than that of the non-polarized state $[15,16]$. In addition, the spin-polarized state becomes unstable with respect to the non-polarized state in the presence of a ballistic current through the GNR [17]. Moreover, the magnetization is theoretically proved to be forbidden in one- and two-dimensional systems at finite temperatures [18]. While most transistors work at a finite temperature (typically room temperature), one can consider only the non-polarized metallic state of ZGNRs for the investigation of ZGNR-based transistors [19,20]. The electronic properties of $\mathrm{B}-\mathrm{C}-\mathrm{N}$ nanostructures and their application in electronic devices have been extensively studied [20-24]. It is shown that a gap can be opened in the GNR band structure by doping with BN [25,26]. This band gap is attributed to the broken symmetry of the graphene sub-lattices [25]. BN-doped ZGNRs may be used as a channel material for nanometer-sized conventional field effect transistors. Although the one-atom thick nature of GNRs makes them highly sensitive to their environmental surroundings, for example, the substrate material, phonons from the substrate and charged impurities, here, we only consider the effect of the substrate material. Any substrate material has some surface roughness (SR). Substrate surface roughness induces a conformal surface roughness on the GNR placed on top of it [27]. It has been shown that SR decreases the mobility of both armchair and zigzag GNRs [28]. Also the performance of armchair GNRFETs (AGNRFETs) is significantly degraded by SR [29]. In this work the device performance of symmetrically BN-doped zigzag graphene nanoribbon field effect transistors (s-BN-ZGNRFETs) is numerically studied for the first time. $\mathrm{SiO}_{2}, \mathrm{BN}$ and mica substrates with SR amplitudes of approximately $168-360,75$ and 24 pm, respectively, are considered [27,30,31]. Rough surfaces are generated by means of Gaussian auto-correlation functions [32,33]. The electronic transport calculations are performed in the framework of a non-equilibrium Green's function and tight-binding Hamiltonian. Because of the statistical nature of SR an ensemble average is taken over a large number of devices to obtain averaged device characteristics.

\section{Model and Methods}

The $\mathrm{sp}^{2}$ hybridization of carbon atoms in the GNR lattice is preserved in the presence of $\mathrm{B}$ or $\mathrm{N}$ dopants [34]. Therefore one can model the electronic structure of BN-doped ZGNRs with a first nearest neighbor tight-binding Hamiltonian [34]. The hopping parameters between $\mathrm{C}, \mathrm{B}$ and $\mathrm{N}$ atoms and the on-site energies in place of $\mathrm{B}$ and $\mathrm{N}$ atoms are set according to [35]. Due to the Gaussian distribution of the experimental data, a two-dimensional Gaussian auto-correlation function is used as a generator of surface roughness [27,30,31]:

$$
\mathrm{C}(\mathbf{r})=\langle\delta h(\mathbf{R}) \delta h(\mathbf{R}+\mathbf{r})\rangle=\delta_{\mathrm{SR}}^{2} \exp \left(-r^{2} / l_{\mathrm{SR}}^{2}\right)
$$

where $h(\mathbf{r})$ is the variation of the surface height at point $\mathbf{r}$. The values $\delta_{\mathrm{SR}}$ and $l_{\mathrm{SR}}=\sqrt{l_{\mathrm{x}}^{2}+l_{\mathrm{y}}^{2}}$ represent the root mean square and correlation length of the height fluctuations, respectively. The $p_{z}$ orbital of carbon is responsible for the electronic properties of graphene. Although the SR modulates both the distance and angle between the $\mathrm{p}_{\mathrm{z}}$ orbitals through, stretching and bending of $\mathrm{C}-\mathrm{C}$ bonds, respectively, the effect of bending is negligible [36]. Therefore, in order to incorporate the effect of the SR into the tight-binding Hamiltonian, the GNR lattice is mapped on the $\mathrm{C}(\mathbf{r})$ surface and the heights of carbon atoms are modulated accordingly. The lattice mismatch of graphene and $\mathrm{BN}$ is $1.7 \%$, which is negligible in comparison to the displacement of atoms due to SR [37]. Therefore, the hopping parameters between displaced atoms of any type can be written as [38,39]:

$$
t(r)=t_{0} \exp \left(-3.37\left(r / r_{0}-1\right)\right)
$$

where $t$ and $t_{0}$ represent the hopping integral between two displaced atoms and the first nearest neighbor hopping parameter of the flat GNR, respectively. All bond lengths of the flat $\mathrm{BN}$-doped ZGNR should be equal to the $\mathrm{C}-\mathrm{C}$ bond length represented by $r_{0}=1.42 \AA$. Having the system Hamiltonian at hand, the retarded Green's function can be written as [40]:

$$
G^{r}(E)=[(E+i \eta) I-H-\Sigma(E)]^{-1}
$$

where $\eta$ is an infinitesimal positive number. The non-Hermitian self-energy matrix, $\Sigma(E)=\Sigma_{\mathrm{S}}(E)+\Sigma_{\mathrm{D}}(E)$, represents the escape rates of electrons from the device into the semi-infinite source and drain ideal leads. The self-energy matrices are calculated through a highly convergent recursive method [41]. The transmission as a function of energy is obtained through the Landauer formula [42]:

$$
T(E)=\operatorname{Tr}\left[\Gamma_{\mathrm{D}}(E) G^{r}(E) \Gamma_{\mathrm{S}}(E) G^{a}(E)\right]
$$

in which $G^{a}(E)=\left[G^{r}(E)\right]^{\dagger}$ is the advanced Green's function and $\Gamma_{i}(i=\mathrm{S}, \mathrm{D})$ represents the level broadening due to the coupling of the device to the leads:

$$
\Gamma_{i}(E)=i\left[\Sigma_{i}(E)+\Sigma_{i}^{\dagger}(E)\right]
$$


Finally, the current through the device is calculated through the Landauer formula $[33,34]$ :

$$
I=\frac{2 q}{h} \int_{-\infty}^{\infty} d E T(E)\left[f_{\mathrm{S}}(E)-f_{\mathrm{L}}(E)\right]
$$

in which $q$ is the electron charge and $f$ represents the Fermi-Dirac distribution function. To shed more light on the physics behind the device performance, the concept of the transport gap has been employed, which is defined as twice the energy in which the transmission drops below $10^{-2}[43,44]$. A more detailed description of the methods is available in [28]. The transistor is considered to be in the on-state when the voltage difference between the gate and the source contacts $\left(V_{\mathrm{GS}}\right)$ equals the maximum supplied voltage to the device $\left(V_{\mathrm{DS}}\right)$, which is considered to be $1 \mathrm{~V}$ herein. The off-state is defined as the device state at $V_{\mathrm{GS}}=0$. The on- and off-current of the device are defined as the current flow from the source to the drain in the on- and off-state, respectively [45].

\section{Results and Discussion}

A typical sample of s-BN-ZGNR with a width of five hexagons of atoms (5h) is shown in Figure 1 with (right) and without (left) surface roughness. As depicted, the one hexagonal chain
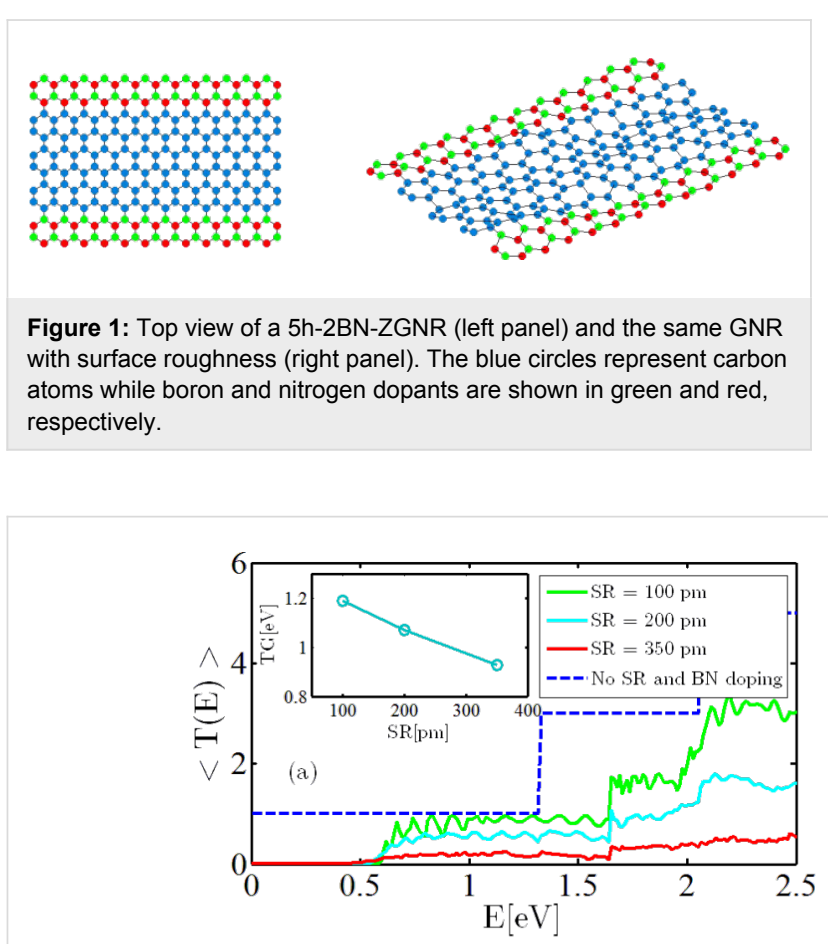

of atoms at each edge along the transport direction is doped with $\mathrm{BN}$ dimers, namely, a 5h-2BN-ZGNR structure.

Figure 2a illustrates the average transmission of a $10 \mathrm{~nm}$ length 4h-2BN-ZGNR versus energy for various SR amplitudes. As shown by increasing the roughness amplitude, the transmission decreases due to an increased SR scattering rate [28]. The inset of Figure $2 \mathrm{a}$ shows that by increasing the roughness amplitude from 100 to $350 \mathrm{pm}$, the BN-doping induced transport gap decreases from 1.19 to $0.99 \mathrm{eV}$ due to decreased average hopping between edge atoms of ZGNR and BN dopants. The average transmission of a $10 \mathrm{~nm}$ length $4 \mathrm{~h}-2 \mathrm{BN}-\mathrm{ZGNR}$ with $200 \mathrm{pm}$ SR amplitude for various correlation lengths is shown in Figure $2 b$. As depicted by decreasing the correlation length, the transmission decreases due to the larger scattering rate of the rougher surface [28]. The transport gap however decreases by decreasing the correlation length (see inset of Figure $2 b$ ). The roughness-dependent behavior of the transport gap in ZGNRs (insets of Figure $2 a$ and Figure $2 b$ ) is in contrast to the undoped AGNRs for which the transport gap remains constant when the roughness parameters are modulated [28].

The structure of the simulated device is depicted in Figure 3. Figure $4 \mathrm{a}$ shows the average transfer characteristics of a $1.5 \times 10 \mathrm{~nm}^{2} 4 \mathrm{~h}-2 \mathrm{BN}-Z$ GNRFET for various substrate materials in logarithmic scale. As shown by increasing the SR amplitude from $25 \mathrm{pm}$ (mica) to $200 \mathrm{pm}$ (average SR amplitude of $\mathrm{a} \mathrm{SiO}_{2}$ substrate) the on-current decreases from 4.4 to $4.2 \mu \mathrm{A}$ while the off-current increases from 0.27 to $1.1 \mathrm{nA}$ (see Figure $4 \mathrm{~b}$ and Figure $4 \mathrm{c}$ ). The observed decrease in the on-current is due to larger SR scattering rate on rougher surfaces while the off-current increases due to the SR-induced decrease in the transport gap, both stated above. Moving from mica to $\mathrm{SiO}_{2}$, both the roughness amplitude and correlation length increase. The former tends to roughen the surface while the latter tends to flatten it. Therefore one can conclude from

Figure 2: (a) Averaged transmission of $10 \mathrm{~nm}$ length $4 \mathrm{~h}-2 \mathrm{BN}-Z \mathrm{GNR}$ as function of the energy for various $\mathrm{SR}$ amplitudes $\left(I_{\mathrm{x}}=I_{\mathrm{y}}=25 \mathrm{~nm}\right)$. The dashed-blue line belongs to the flat channel (a 4h-2BN-ZGNR without SR). The inset shows the transport gap versus SR amplitude. (b) Averaged transmission probability of $10 \mathrm{~nm}$ length $4 \mathrm{~h}-2 \mathrm{BN}-\mathrm{ZGNR}$ as function of the energy for various SR correlation lengths $\left(\delta_{\mathrm{SR}}=200 \mathrm{pm}\right)$. The inset shows the transport gap versus SR correlation length. 
Figure $4 \mathrm{~b}-\mathrm{d}$ that for the substrate materials selected for this work the SR amplitude effect dominates that of the correlation length. Figure $4 \mathrm{~d}$ shows that the on/off-current ratio for a $10 \mathrm{~nm}$ long 4h-2BN-ZGNRFET on a $\mathrm{SiO}_{2}$ substrate does not meet the required amount of $10^{4}$ for digital transistors [5]. It is possible however to improve the device performance in terms of on/offcurrent ratio by increasing the width of the $\mathrm{BN}$-doped regions.

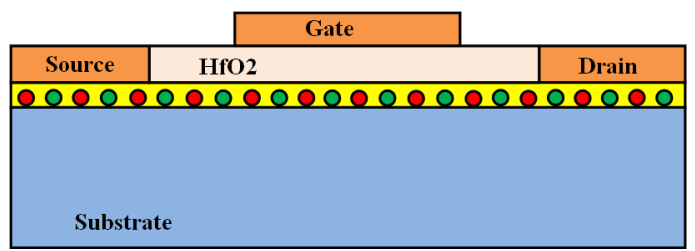

Figure 3: Schematic representation of the simulated device structure. The gate insulator is assumed to be $2.5 \mathrm{~nm}$ thick $\mathrm{HfO}_{2}(\mathrm{~K}=25)$. Source and drain contacts are heavily doped extensions of the channel without SR.
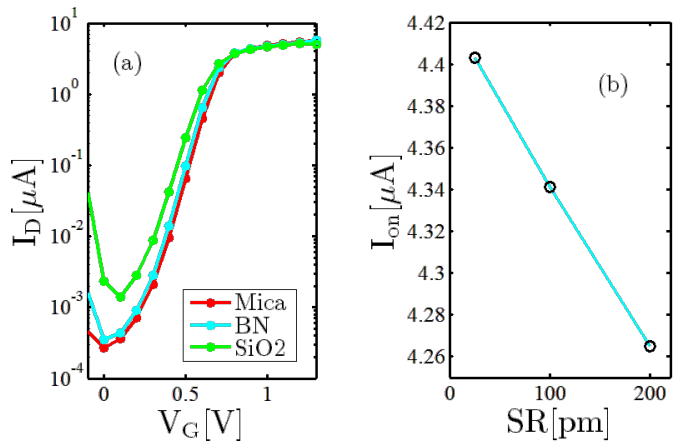

Figure 5a shows the average transfer characteristics of two $10 \mathrm{~nm}$ length devices with different device widths but the same $\mathrm{BN}$ widths (3h-2BN and $5 \mathrm{~h}-2 \mathrm{BN})$ with (red) and without (green) SR. As shown, by increasing the width from 1.14 to $2 \mathrm{~nm}$, both the on- and the off-current increase. The on-current increases due to an increased number of conducting channels while the observed increase in the off-current is due to decreased HOMO-LUMO gap (and, consequently, a decreased transport gap). As a result of this, the on/off-current ratio decreases from $1.6 \times 10^{6}$ in the $3 \mathrm{~h}-2 \mathrm{BN}$ device to 156 in the $5 \mathrm{~h}-2 \mathrm{BN}$ device (see inset of Figure $5 \mathrm{a}$ ). To resolve the problem of an increased off-current in wider devices, the width of the $\mathrm{BN}$-doped region must be increased. Figure $5 \mathrm{~b}$ shows the average transfer characteristic of a $2.84 \times 10 \mathrm{~nm}^{2}$ device $(7 \mathrm{~h})$ with a $\mathrm{SiO}_{2}$ substrate for various $\mathrm{BN}$ widths. By doping the three hexagonal chain from each edge of the ZGNR (7h-6BNZGNRFET) the on/off-current ratio reaches $4.86 \times 10^{4}$, which falls within the acceptable range for digital transistors (see Figure 5c).

Figure 4: (a) Averaged transfer characteristic (b) on-current (c) off-current and (d) on-/off-current ratio of a $1.56 \times 10 \mathrm{~nm} \mathrm{n}^{2} 4 \mathrm{~h}-2 \mathrm{BN}-\mathrm{ZGNRFET}$ for various substrate materials. In panels (b) to (d) the horizontal axis shows the $\mathrm{SR}$ amplitude. The correlation length is considered to be $25 \mathrm{~nm}$ for $\mathrm{SiO}_{2}$ and $5 \mathrm{~nm}$ for mica and $\mathrm{BN}[27,30,31]$.
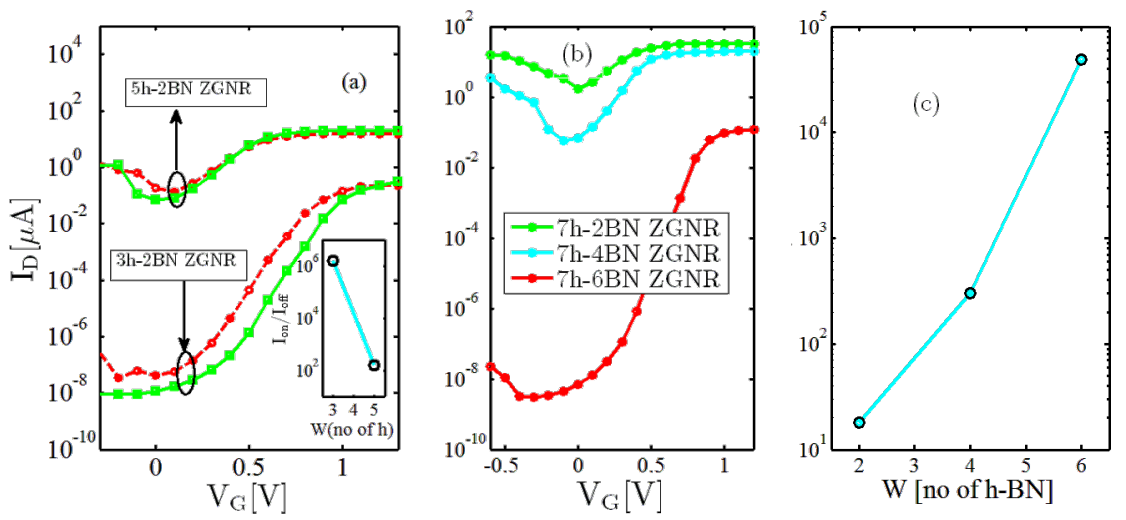

Figure 5: (a) The average transfer characteristic of $10 \mathrm{~nm}$ length devices with $\mathrm{SiO}_{2}$ substrate for different device widths but the same $\mathrm{BN}$ widths $(3 \mathrm{~h}$ 2BN and $5 \mathrm{~h}-2 \mathrm{BN}$ ) with (red) and without (green) SR. The inset shows the on-/off-current ratio of rough devices with respect to device width. (b) The average transfer characteristic and (c) the on-/off-current ratio of a $2.84 \times 10 \mathrm{~nm}^{2}$ device $(7 \mathrm{~h}) \mathrm{with}_{\mathrm{SiO}_{2}}$ substrate for various $\mathrm{BN}$ widths. 


\section{Conclusion}

In summary, symmetrical BN doping was applied on ZGNRs to open a necessary gap for use in digital electronic applications. Next, the device performance of FETs with symmetrically BN-doped ZGNRs as a channel material was numerically studied. Effect of the surface roughness for several common substrate materials on the device performance was explored. To achieve the minimum on/off-current ratio of $10^{4}$ for s-BNZGNRFETs, an appropriate substrate material must be chosen. In general it is possible to widen the device and the BN-doped portion of the channel to achieve larger a on/off-current ratio. Results show that by symmetrical doping ZGNRs with BN and appropriate selection of substrate material in terms of the surface roughness, ZGNRFETs can meet the required on/offcurrent ratio for future digital electronics.

\section{References}

1. ITRS The International Technology Roadmap for Semiconductors. http://www.itrs.net (accessed June 2, 2014).

2. Banerjee, S. K.; Register, L. F.; Tutuc, E.; Basu, D.; Kim, S.; Reddy, D.; MacDonald, A. H. Proc. IEEE 2010, 98, 2032-2046. doi:10.1109/JPROC.2010.2064151

3. Novoselov, K. S.; Geim, A. K.; Morozov, S. V.; Jiang, D.; Zhang, Y.; Dubonos, S. V.; Grigorieva, I. V.; Firsov, A. A. Science 2004, 306, 666-669. doi:10.1126/science.1102896

4. Berger, C.; Song, Z.; Li, T.; Li, X.; Ogbazghi, A. Y.; Feng, R.; Dai, Z.; Marchenkov, A. N.; Conrad, E. H.; First, P. N.; de Heer, W. A. J. Phys. Chem. B 2004, 108, 19912-19916. doi:10.1021/jp040650f

5. Schwierz, F. Proc. IEEE 2013, 101, 1567-1584. doi:10.1109/JPROC.2013.2257633

6. Kim, K.; Choi, J.-Y.; Kim, T.; Cho, S.-H.; Chung, H.-J. Nature 2011, 479, 338-344. doi:10.1038/nature10680

7. Ezawa, M. Phys. Rev. B 2006, 73, 045432. doi:10.1103/PhysRevB.73.045432

8. Son, Y.; Cohen, M. L.; Louie, S. G. Phys. Rev. Lett. 2006, 97, 216803. doi:10.1103/PhysRevLett.97.216803

9. Barone, V.; Hod, O.; Scuseria, G. E. Nano Lett. 2006, 6, 2748. doi:10.1021/nI0617033

10. Liu, L.; Shen, Z. Appl. Phys. Lett. 2009, 95, 252104. doi:10.1063/1.3276068

11. Chen, Z.; Lin, Y.-M.; Rooks, M. J.; Avouris, P. Physica E 2007, 40, 228-232. doi:10.1016/j.physe.2007.06.020

12. Fujita, M.; Wakabayashi, K.; Nakada, K.; Kusakabe, K. J. Phys. Soc. Jpn. 1996, 65, 1920. doi:10.1143/JPSJ.65.1920

13. Nakada, K.; Fujita, M.; Dresselhaus, G.; Dresselhaus, M. S. Phys. Rev. B 1996, 54, 17954. doi:10.1103/PhysRevB.54.17954

14. Li, Y.; Zhou, Z.; Shen, P.; Chen, Z. ACS Nano 2009, 3, 1952. doi:10.1021/nn9003428

15. Son, Y.-W.; Cohen, M. L.; Louie, S. G. Nature 2006, 444, 347. doi:10.1038/nature05180

16. Wu, F.; Kan, E.; Xiang, H.; Wei, S.-H.; Whangbo, M.-H.; Yang, J. Appl. Phys. Lett. 2009, 94, 223105. doi:10.1063/1.3147854

17. Areshkin, D. A.; White, C. T. Nano Lett. 2007, 11, 3253. doi:10.1021/nl070708c

18. Mermin, N. D.; Wagner, H. Phys. Rev. Lett. 1966, 17, 1133. doi:10.1103/PhysRevLett.17.1133
19. Li, Z.; Qian, H.; Wu, J.; Gu, B.-L.; Duan, W. Phys. Rev. Lett. 2008, 100, 206802. doi:10.1103/PhysRevLett.100.206802

20. Huang, B.; Yan, Q.; Zhou, G.; Wu, J.; Gu, B.-L.; Duan, W.; Liu, F. Appl. Phys. Lett. 2007, 91, 253122. doi:10.1063/1.2826547

21. Martins, T. B.; Miwa, R. H.; da Silva, A. J. R.; Fazzio, A. Phys. Rev. Lett. 2007, 98, 196803. doi:10.1103/PhysRevLett.98.196803

22. Yamijala, S. SRKC.; Bandyopadhyay, A.; Pati, S. K. J. Phys. Chem. C 2013, 117, 23295-23304. doi:10.1021/jp406344z

23. Kaneko, T.; Harigaya, K.; Imamura, H. J. Phys. Soc. Jpn. 2013, 82, 083710. doi:10.7566/JPSJ.82.083710

24. Fiori, G.; Betti, A.; Bruzzone, S.; lannaccone, G. ACS Nano 2012, 6 , 2642-2648. doi:10.1021/nn300019b

25. Shinde, P. P.; Kumar, V. Phys. Rev. B 2011, 84, 125401. doi:10.1103/PhysRevB.84.125401

26. Fan, X.; Shen, Z.; Liu, A. Q.; Kuo, J.-L. Nanoscale 2012, 4, 2157. doi:10.1039/c2nr11728b

27. Geringer, V.; Liebmann, M.; Echtermeyer, T.; Runte, S.; Schmidt, M.; Rückamp, R.; Lemme, M. C.; Morgenestern, M. Phys. Rev. Lett. 2009, 102, 076102. doi:10.1103/PhysRevLett.102.076102

28. Touski, S. B.; Pourfath, M. Appl. Phys. Lett. 2013, 103, 143506. doi:10.1063/1.4824362

29. Sanaeepur, M.; Goharrizi, A. Y.; Sharifi, M. J. IEEE Trans. Electron Devices 2014, 61, 1193-1198. doi:10.1109/TED.2013.2290049

30. Dean, C. R.; Young, A. F.; Meric, I.; Lee, C.; Wang, L.; Sorgenfrei, S.; Watanabe, K.; Taniguchi, T.; Kim, P.; Shepard, K. L.; Hone, J. Nat. Nanotechnol. 2010, 5, 722-726. doi:10.1038/nnano.2010.172

31. Lui, C. H.; Liu, L.; Mak, K. F.; Flynn, G. W.; Heinz, T. F. Nature 2009, 462, 339-341. doi:10.1038/nature08569

32. Wu, J.-J. Tribol. Int. 2000, 33, 47-58. doi:10.1016/S0301-679X(00)00016-5

33. Goharrizi, A.-Y.; Pourfath, M.; Fathipour, M.; Kosina, H. IEEE Trans. Electron Devices 2012, 59, 3527-3532. doi:10.1109/TED.2012.2218817

34. Marconcini, P.; Cresti, A.; Triozon, F.; Fiori, G.; Biel, B.; Niquet, Y.-M.; Macucci, M.; Roche, S. ACS Nano 2012, 6, 7942-7947. doi:10.1021/nn3024046

35. Zhao, R.; Wang, J.; Yang, M.; Liu, Z.; Liu, Z. J. Phys. Chem. C 2012, 116, 21098-21103. doi:10.1021/jp306660x

36. Klos, J. W.; Shylau, A. A.; Zozulenko, I. V.; Xu, H.; Heinzel, T. Phys. Rev. B 2009, 80, 245432. doi:10.1103/PhysRevB.80.245432

37. Mukherjee, S.; Kaloni, T. P. J. Nanopart. Res. 2012, 14, 1059-1063. doi:10.1007/s11051-012-1059-2

38. Pereira, V. M.; Castro Neto, A. H.; Peres, N. M. R. Phys. Rev. B 2009, 80, 045401. doi:10.1103/PhysRevB.80.045401

39. Castro Neto, A. H.; Guinea, F. Phys. Rev. B 2007, 75, 045404. doi:10.1103/PhysRevB.75.045404

40. Datta, S. Quantum transport: from atoms to transistors, 2nd ed.; Cambridge University Press: Cambridge, 2005.

41. Lopez Sancho, M. P.; Lopez Sancho, J. M.; Sancho, J. M. L.; Rubio, J. J. Phys. F: Met. Phys. 1985, 15, 851. doi:10.1088/0305-4608/15/4/009

42. Anantram, M. P.; Svizhenko, A. IEEE Trans. Electron Devices 2007, 54, 2100-2115. doi:10.1109/TED.2007.902857

43. Han, M. Y.; Özyilmaz, B.; Zhang, Y.; Kim, P. Phys. Rev. Lett. 2007, 98, 206805-206808. doi:10.1103/PhysRevLett.98.206805

44. Cresti, A.; Roche, S. New J. Phys. 2009, 11, 095004. doi:10.1088/1367-2630/11/9/095004

45. Schwierz, F. Nat. Nanotechnol. 2010, 5, 487-496. doi:10.1038/nnano.2010.89 


\section{License and Terms}

This is an Open Access article under the terms of the Creative Commons Attribution License

(http://creativecommons.org/licenses/by/2.0), which permits unrestricted use, distribution, and reproduction in any medium, provided the original work is properly cited.

The license is subject to the Beilstein Journal of Nanotechnology terms and conditions:

(http://www.beilstein-journals.org/bjnano)

The definitive version of this article is the electronic one which can be found at:

doi:10.3762/bjnano.5.168 